\title{
U-SPECT-II: An Ultra-High-Resolution Device for Molecular Small-Animal Imaging
}

Frans van der Have*1,2, Brendan Vastenhouw ${ }^{* 1,2}$, Ruud M. Ramakers ${ }^{1}$, Woutjan Branderhorst ${ }^{1}$, Jens O. Krah ${ }^{3}$, Changguo Ji ${ }^{1}$, Steven G. Staelens ${ }^{4}$, and Freek J. Beekman ${ }^{1,2,5}$

${ }^{I}$ Image Sciences Institute, University Medical Center Utrecht, Utrecht, The Netherlands; ${ }^{2}$ MILabs B.V., Utrecht, The Netherlands; ${ }^{3}$ Institute of Telecommunications Technology, Cologne University of Applied Sciences, Cologne, Germany; ${ }^{4}$ Ghent University-IBBT, Ghent, Belgium; and ${ }^{5}$ Delft University of Technology, Delft, The Netherlands

We present a new rodent SPECT system (U-SPECT-II) that enables molecular imaging of murine organs down to resolutions of less than half a millimeter and high-resolution total-body imaging. Methods: The U-SPECT-II is based on a triangular stationary detector set-up, an $X Y Z$ stage that moves the animal during scanning, and interchangeable cylindric collimators (each containing 75 pinhole apertures) for both mouse and rat imaging. A novel graphical user interface incorporating preselection of the field of view with the aid of optical images of the animal focuses the pinholes to the area of interest, thereby maximizing sensitivity for the task at hand. Images are obtained from list-mode data using statistical reconstruction that takes system blurring into account to increase resolution. Results: For $99 \mathrm{mTc}$, resolutions determined with capillary phantoms were smaller than 0.35 and $0.45 \mathrm{~mm}$ using the mouse collimator with 0.35 - and $0.6-\mathrm{mm}$ pinholes, respectively, and less than $0.8 \mathrm{~mm}$ using the rat collimator with $1.0-\mathrm{mm}$ pinholes. Peak geometric sensitivity is $0.07 \%$ and $0.18 \%$ for the mouse collimator with 0.35 - and $0.6-\mathrm{mm}$ pinholes, respectively, and $0.09 \%$ for the rat collimator. Resolution with

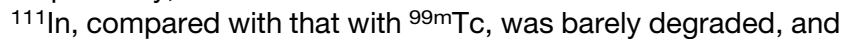
resolution with ${ }^{125}$ I was degraded by about $10 \%$, with some additional distortion. In vivo, kidney, tumor, and bone images illustrated that U-SPECT-II could be used for novel applications in the study of dynamic biologic systems and radiopharmaceuticals at the suborgan level. Conclusion: Images and movies obtained with U-SPECT-II provide high-resolution radiomolecule visualization in rodents. Discrimination of molecule concentrations between adjacent volumes of about $0.04 \mu \mathrm{L}$ in mice and $0.5 \mu \mathrm{L}$ in rats with U-SPECT-II is readily possible.

Key Words: molecular imaging; pinhole; SPECT; small animal; reconstruction

J Nucl Med 2009; 50:599-605

DOI: 10.2967/jnumed.108.056606

B iomedical research benefits from quantitative in vivo techniques and the ability to perform longitudinal studies in individual animals. Using radiolabeled molecules effec-

Received Aug. 3, 2008; revision accepted Dec. 24, 2008.

For correspondence or reprints contact: Freek J. Beekman, Delft University of Technology, Faculty of Applied Sciences, Department of Radiation, Radionuclides and Medical Imaging, Mekelweg 15, NL 2629 BJ, Delft, The Netherlands.

E-mail: f.j.beekman@tudelft.nl

${ }^{*}$ Contributed equally to this work.

COPYRIGHT () 2009 by the Society of Nuclear Medicine, Inc. tively in rodent models requires dedicated SPECT and PET instruments. For a more beneficial resolution-sensitivity trade-off, most dedicated small-animal SPECT systems use pinhole collimation. Introductions to the subject of pinhole SPECT can be found in studies by King et al. (1), Meikle et al. (2), and Beekman and van der Have (3).

To enable proper tomographic image reconstruction, projections of the object must be available from a sufficient number of different angles. Most SPECT systems achieve this by rotating either the detector or the object (4-9). Stationary small-animal pinhole SPECT systems (10-13) avoid the need for rotation by using detector set-ups that cover $360^{\circ}$ and many pinholes to provide a large number of angles under which the animal is observed. These systems have fewer moving parts and are, therefore, less prone to mechanical problems and the need for time-consuming calibration procedures. They also provide the advantage of enabling dynamic imaging with arbitrarily short frame lengths $(11,14,15)$. The full $360^{\circ}$ coverage, in combination with many focusing micropinholes and a high magnification factor to maximize the information content per photon, provides a reconstructed image resolution that can be significantly better than that of state-of-the-art small-animal PET $(3,16)$.

The aim of our present work was to describe a dedicated and user-friendly ultra-high-resolution multipinhole SPECT scanner (U-SPECT-II; MILabs; Fig. 1A) suitable for dynamic imaging of small experimental animals such as rats and mice. The system is equipped with unique tools to maximize focusing capabilities and the associated sensitivity when imaging a part of the animal. This and other new features make U-SPECT-II a significant improvement over its predecessor (U-SPECT-I) $(12,14,17)$. In the present work, we characterized the performance of U-SPECT-II on sensitivity and resolution for different mouse and rat collimators using phantom experiments. Animal scans demonstrate the variety of unique imaging capabilities of the U-SPECT-II.

\section{MATERIALS AND METHODS}

\section{System Description}

With U-SPECT-II, different multipinhole collimators, together with shielding parts to prevent overlap, are easily exchangeable to 

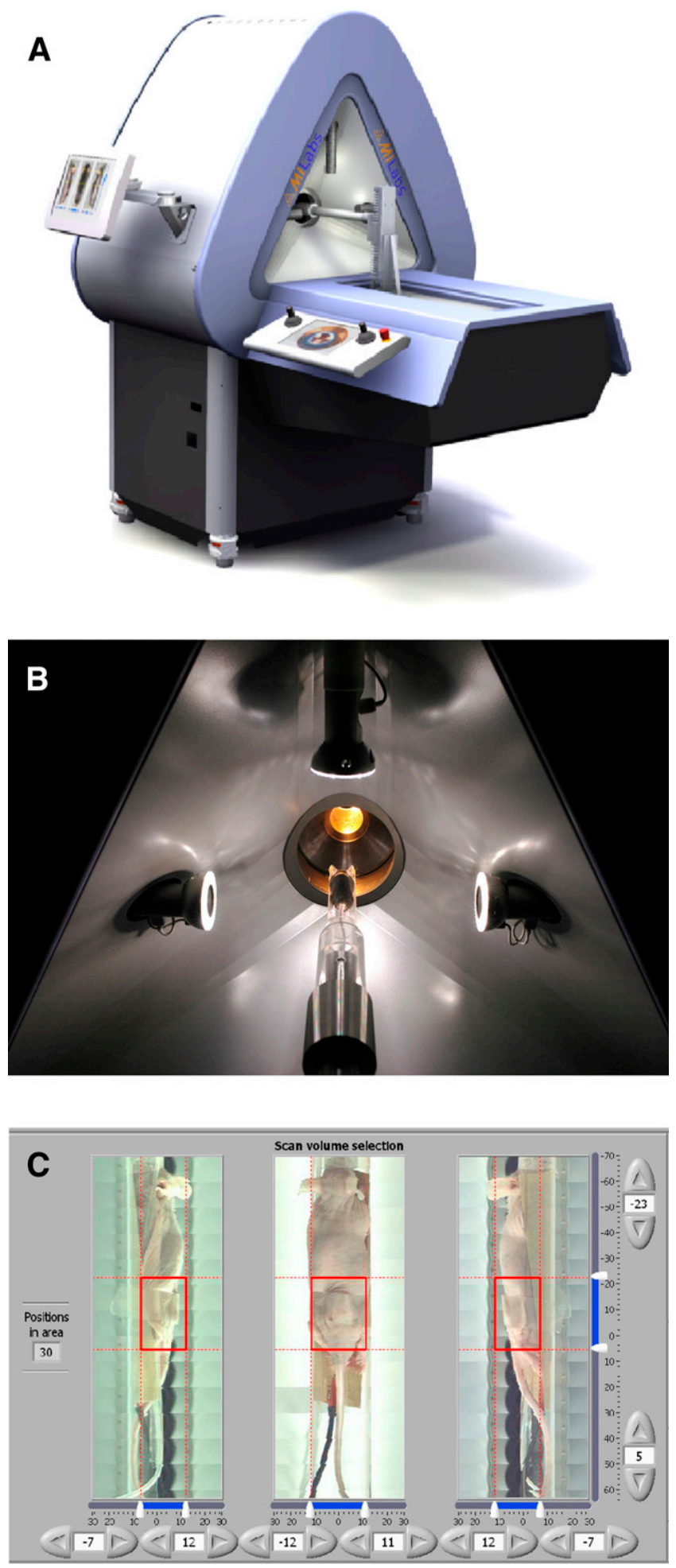

FIGURE 1. (A) U-SPECT-II system. (B) Mouse observed with 3 optical cameras for ROI selection. (C) User interface for selection of $\mathrm{ROI}$, for example, a tumor. $\mathrm{ROI}$ = region of interest.

facilitate optimal imaging for animals with different sizes, scanning times, and tracer doses. The collimator tubes (diameters, 44 and 98 $\mathrm{mm}$ ) are optimized for mouse- or rat-sized animals, respectively. Except for an $X Y Z$ stage that moves the bed, the scanner is stationary; that is, there is no need to move the collimator, detectors, or animal when a specific organ that fits inside the field of view (FOV) is being imaged. If a larger volume of interest is desired (e.g., in total-body imaging), then the animal bed is translated into 3 dimensions using the $X Y Z$ stage. For most cases, this translation can be done quickly enough to still enable a combination of multipleposition acquisitions and dynamic imaging at a time scale of tens of seconds up to a few minutes. Large scintillation detectors that are divided into nonoverlapping cameras are used, allowing a high resolution and high sensitivity to be obtained at the same time. Listmode acquisition provides flexibility in processing the data afterward, for example, for retrospective (multiple) gating, choice of time frame length, and selection of energy windows for single- and multiple-isotope imaging and scatter correction.

The collimators presented here all consist of a tungsten cylinder with 5 rings of 15 pinhole apertures. Pinhole positions in each ring are rotated with respect to the pinhole positions in adjacent rings, to increase angular sampling of the object. All pinholes focus on a single volume (i.e., the central FOV) in the center of the tube. The collimators are surrounded with tungsten shielding that prevents the projections on the detector from overlapping. The division of the detector in mini- $\gamma$-cameras can be determined by placing a bottle with uniformly distributed activity that is larger than the FOV in the collimator tube and acquiring the projection image (12).

Switches automatically distinguish which of the collimators and beds are mounted, and the motion of the translation stage is restricted to prevent collisions. The mounting mechanism of the bed to the robot is made such that the bed is locked in place and can be unlocked and transferred to another scanner, for example, to a micro-CT scanner, while keeping the animal in position. This approach $(18,19)$, combined with a calibration phantom, can be used to obtain registered images from independent imaging modalities without the use of fiducial markers.

Before being moved into the scanner, the animal is placed between 3 optical cameras that acquire images from the 2 sides and from the top (Fig. 1B). In the side and top views, users can select which area (volume) needs to be scanned by moving sliders (Fig. 1C). The software of the system then calculates the sequence of positions necessary to acquire data from this volume and automatically moves the animal through the focus during the acquisition, using this sequence (17). The selection of the region of interest in the graphical user interface (20) makes the system as a whole user-friendly.

Images were reconstructed using an ordered-subset algorithm and a precalculated system matrix (16).

Table 1 compares features of U-SPECT-II with its predecessor, U-SPECT-I. One notable difference is the much larger surface area of the detectors. The other design parameters were chosen such that the peak sensitivity and resolution were approximately the same in U-SPECT-II as they were in U-SPECT-I. The FOV seen in a single position is significantly larger, however, effectively increasing the sensitivity in multiposition scans. Important other differences include list-mode data availability, retrospective dual-gating availability, and graphical user interface plus webcambased navigation of pinhole focus.

In U-SPECT-II, the signal of each individual photomultiplier tube is digitized, and the electronics in the camera heads, together with the device driver code in the acquisition computer, apply linearity, energy, and uniformity correction. The detected events are sent to the acquisition computer via a gigabit Ethernet connection and stored in a list-mode format containing the horizontal and vertical position (12 bits), energy, and a time stamp for each event. 
TABLE 1. Comparison of U-SPECT-I with U-SPECT-II

\begin{tabular}{|c|c|c|}
\hline Parameter & U-SPECT-I & U-SPECT-II \\
\hline \multicolumn{3}{|l|}{ Detectors } \\
\hline Crystal length $\times$ width $\times$ thickness $(\mathrm{mm})$ & $410 \times 250 \times 9.5$ & $508 \times 381 \times 9.5$ \\
\hline No. of detectors & 3 & 3 \\
\hline No. of PMTs per detector & 49 & 55 \\
\hline PMT readout method & Analog, resistor network & Each PMT signal digitized \\
\hline Data collection & Planar pixel image & List-mode data \\
\hline \multicolumn{3}{|l|}{ Pinhole collimator configuration } \\
\hline No. of rings and pinholes per ring & $5 \times 15$ & $5 \times 15$ \\
\hline Mouse single-bed position FOV diameter $(\mathrm{mm})$ & 10.5 & 12 \\
\hline Mouse single-bed position FOV axial (mm) & 5 & 7 \\
\hline Rat single-bed position FOV diameter (mm) & N/A & 27 \\
\hline Rat single-bed position FOV axial (mm) & N/A & 11 \\
\hline Mouse collimator bore diameter (mm) & 39 & 44 \\
\hline Mouse radial position of pinholes (mm) & 22 & 24 \\
\hline Rat collimator bore diameter $(\mathrm{mm})$ & N/A & 98 \\
\hline Rat radial position of pinholes (mm) & N/A & 53 \\
\hline Mouse pinhole material and diameters (mm) & Gold, 0.3, 0.6 & Gold, $0.35,0.6$ \\
\hline Rat pinhole material and diameters $(\mathrm{mm})$ & N/A & Tungsten, $0.7-1.5$, gold optional \\
\hline Mouse pinhole tube material & Tungsten & Tungsten \\
\hline Mouse shielding tube material & Lead & Tungsten \\
\hline Rat collimator tube material & N/A & Tungsten \\
\hline \multicolumn{3}{|l|}{ General features } \\
\hline Mouse collimator, 0.6-mm-pinhole peak efficiency (\%) & 0.22 & 0.18 \\
\hline Mouse collimator, 0.35-mm-pinhole peak efficiency (\%) & 0.07 & 0.07 \\
\hline Rat collimator, 1.0-mm-pinhole peak efficiency (\%) & N/A & 0.09 \\
\hline Gating possible & No & Yes, multiple \\
\hline Dead time between measurements (s) & 12 & $<2$ \\
\hline Dynamic sequence imaging & Manual start & Automatic \\
\hline Coordination of scan sequence with acquisition & Manual & Automatic \\
\hline GUI-based navigator available & No & Yes \\
\hline
\end{tabular}

The animal is moved stepwise through the pinhole focus during acquisition. We developed a method to take the data from all bed positions into account simultaneously during reconstruction. This is an improvement over reconstructing each animal position separately and subsequently stitching together partial reconstructions (17).

\section{Performance Characterization}

The sensitivity of the system along the axial and transaxial directions was measured by scanning a ${ }^{99 \mathrm{~m}} \mathrm{Tc}$ point source of known activity through the center of the FOV.

The reconstructed spatial resolution was characterized by using capillary resolution phantoms. For the testing of the mouse collimator tubes (pinhole diameters, 0.35 and $0.6 \mathrm{~mm}$ ), the capillary diameters used in the 6 different segments were $0.25,0.3,0.35,0.4$, 0.5 , and $0.6 \mathrm{~mm}$, the distances between capillaries in a segment equal to the capillary diameter within that segment. For the rat collimator, the diameters were $0.7,0.8,0.9,1.0,1.2$, and $1.5 \mathrm{~mm}$. The phantoms were scanned for $2 \mathrm{~h}$ with a ${ }^{99 \mathrm{~m}} \mathrm{Tc}$ concentration of $600 \mathrm{MBq} / \mathrm{mL}$. To compare the resolution performance of the system for different isotopes, the small phantom was filled with a concentration of ${ }^{125} \mathrm{I},{ }^{99 \mathrm{~m}} \mathrm{Tc}$, and ${ }^{111} \mathrm{In}(74 \mathrm{MBq} / \mathrm{mL})$ and subsequently scanned for $2 \mathrm{~h}$ in the $0.6-\mathrm{mm}$-pinhole mouse collimator.

\section{Initial Animal Experiments}

Animal studies were conducted following protocols approved by the Animal Research Committee of the University Medical Center
Utrecht and the University of Ghent. To emulate either a proportionally shorter scan time or a lower activity without having to scan more animals, some of the datasets were reconstructed using the full scan time and also $10 \%$, or even $1 \%$, of the counts from the list-mode data. This method saves animals and allows a comparison of images of different acquisition times or doses with the animal in the same position.

\section{Rat Bone Scan}

A $279-\mathrm{g}$ male Wistar rat was injected with ${ }^{99 m}$ Tc-hydroxymethylene diphosphonate ( $\left.{ }^{99 \mathrm{~m}} \mathrm{Tc}-\mathrm{HDP}\right)(1,740 \mathrm{MBq})$ and anesthetized with isoflurane anesthesia. At $3 \mathrm{~h} 20 \mathrm{~min}$ after radioligand injection, the rat was imaged for $1 \mathrm{~h}$ in the U-SPECT-II scanner using the $1.0-\mathrm{mm}$-diameter pinhole rat collimator tube.

\section{Mouse Kidney Scan}

A male 30-g C57BL/6 mouse was anesthetized with isoflurane and injected intravenously with ${ }^{99 \mathrm{~m}} \mathrm{Tc}$-dimercaptosuccinic acid (DMSA) (138 MBq). At $1 \mathrm{~h}$ after injection, the mouse was scanned for $1 \mathrm{~h}$ in the U-SPECT-II scanner using the 0.35-mm-pinhole mouse collimator tube. This scan was reconstructed both using all of the data (100\%) and using only $10 \%$ of the counts.

\section{Mouse Tumor Imaging}

A 27-g athymic mouse was inoculated to have a 0.5-g Capan-1 pancreatic tumor in the thigh. The animal was injected via the tail vein with $22.2 \mathrm{MBq}$ of ${ }^{111} \mathrm{In}$-diethylenetriaminepentaacetic acid 
(DTPA)-14C5 (14C5 is a monoclonal antibody that is overexpressed on the [pancreatic] tumor surface). At $4 \mathrm{~d}$ after injection (the maximal uptake is at $72 \mathrm{~h}$ ), the animal was anesthetized with isoflurane and imaged for $30 \mathrm{~min}$ using the 0.6-mm-pinhole mouse collimator. The animal was sacrificed afterward, and the tumor was surgically removed.

\section{RESULTS}

\section{Sensitivity Measurements}

For ${ }^{99 \mathrm{~m}} \mathrm{Tc}$, the system sensitivity is shown in Table 2 for the mouse collimator with 0.6 - and $0.35-\mathrm{mm}$ pinholes and for the rat collimator with $1.0-\mathrm{mm}$ pinholes. The values are expressed in counts per second (cps) per $\mathrm{MBq}$ for ${ }^{99 \mathrm{~m}} \mathrm{Tc}$ and as geometric efficiency (excluding capture efficiency and isotope-specific photopeak prevalence).

\section{Resolution Measurements}

Figure 2 shows reconstructions of the capillary phantom to demonstrate the spatial resolution. The top row in Figure 2 shows the maximum achievable resolution for each of the 3 collimators, using a ${ }^{99 \mathrm{~m}} \mathrm{Tc}$ concentration of $600 \mathrm{MBq} / \mathrm{mL}$. The bottom row in Figure 2 shows the difference between the isotopes ${ }^{125} \mathrm{I},{ }^{99} \mathrm{~m} \mathrm{Tc}$, and ${ }^{111} \mathrm{In}$ in the mouse collimator with 0.6-mm pinholes. The concentration for the bottom row was $74 \mathrm{MBq} / \mathrm{mL}$ for each isotope, and the scan time was $2 \mathrm{~h}$. The smallest readily discernable rod sizes for the collimators in the top row are $0.35,0.4$, and $0.8 \mathrm{~mm}$. At least some of the $0.35-\mathrm{mm}$ rods are still visible with the $0.6-\mathrm{mm}$ pinholes, and some of the $0.3-\mathrm{mm}$ rods are visible with the $0.35-\mathrm{mm}$ pinholes. The rod visibility for ${ }^{111}$ In is slightly worse than that for ${ }^{99 \mathrm{~m}} \mathrm{Tc}$. For ${ }^{125} \mathrm{I}$, the rod visibility is worse but still around $0.5 \mathrm{~mm}$. Because the resolution for ${ }^{99 \mathrm{~m}} \mathrm{Tc}$ under these conditions is about $0.45 \mathrm{~mm}$, we estimate the resolution loss due to the lower energy of ${ }^{125} \mathrm{I}$ photons to be about $10 \%$. For ${ }^{125} \mathrm{I}$, some additional distortion in the rods is observed. Because the system was calibrated with a ${ }^{99 \mathrm{~m}} \mathrm{Tc}$ point source without correcting the system matrix for other isotopes, a lower resolution could have been expected. The low photon energy $\left({ }^{125} \mathrm{I}\right)$ results in a poorer detector resolution and more object scatter, and a higher energy $\left({ }^{111} \mathrm{In}\right)$ results in more penetration and collimator scatter.

\section{Initial Animal Experiments}

Rat Bone Scan. Rat HDP data were reconstructed using $100 \%, 10 \%$, and $1 \%$ of the available counts, emulating an injected activity of $1,740 \mathrm{MBq}$ and scan times of approximately 60, 6, and $36 \mathrm{~s}$, respectively. Alternatively, scan times of $1 \mathrm{~h}$ and injected activities of approximately 1,740, 174, and

\begin{tabular}{|c|c|c|}
\hline Collimator & $\begin{array}{l}\text { FOV sensitivity } \\
\text { (cps/MBq }{ }^{99 m T c)}\end{array}$ & $\begin{array}{l}\text { FOV geometric } \\
\text { sensitivity (\%) }\end{array}$ \\
\hline Mouse, 0.6-mm pinhole & 1,500 & 0.18 \\
\hline Mouse, $0.35-\mathrm{mm}$ pinhole & 525 & 0.07 \\
\hline Rat, $1.0-\mathrm{mm}$ pinhole & 700 & 0.09 \\
\hline
\end{tabular}

$17 \mathrm{MBq}$ were emulated. As in the case of the mouse bone scan, the reconstructions were postsmoothed, with an increasing gaussian kernel size to deal with the noise (kernel full width at half maximum, 0.3, 0.66, and $1.43 \mathrm{~mm}$ ).

Figure 3 shows maximum-intensity projections of the 3 reconstructions. The system sensitivity of the $1.0-\mathrm{mm}$ rat collimator tube lies between the sensitivities of the 0.6- and $0.35-\mathrm{mm}$ mouse collimators. The size of the tube has been designed to be more than twice as large as the mouse collimator, at the cost of resolution. Relative to the size of the animal, approximately the same level of anatomic detail is visible in the mouse as is visible in the rat. The rat bone scan was obtained with respiratory gating enabled (12 gates). A movie showing the respiratory motion is available as Supplemental Video 1 (supplemental materials are available online only at http://jnm.snmjournals.org). In addition to monitoring bone turnover, such images make it possible to investigate body motion during respiration and to assess the effect of (or even to correct for the unwanted effect of) respiratory motion on the image sharpness.

Mouse Kidney Imaging. Figure 4 shows slices of a ${ }^{99 \mathrm{~m}} \mathrm{Tc}-$ DMSA image of the right kidney of the mouse. The slice thickness is only $0.375 \mathrm{~mm}$. The uptake in the cortex of the kidney demonstrates the submillimeter resolution of the instrument in this in vivo image. Supplemental Figure 1 shows the same study as the one shown in Figure 4, but the image was reconstructed using only $10 \%$ of the recorded photons. The use of $10 \%$ of the data emulates a scan time of $1 \mathrm{~h}$ with an injected activity of $13.8 \mathrm{MBq}$, or alternatively, a scan time of $6 \mathrm{~min}$ with an injected activity of $138 \mathrm{MBq}$. DMSA is retained for a long time in the cortex of the kidney, especially in the tubules. The images show the distribution of functioning kidney tissue. Such scans can be used to localize defects in the parenchyma or to assess the relative contribution of each kidney, for example, the function of the left versus the right kidney. The resolution in the presented images is sufficient to assess function of tiny parts of mouse kidneys.

Mouse ${ }^{111}$ In Tumor Imaging. Figure 5 shows that USPECT-II delivers good resolution for the medium-energy isotope ${ }^{111} \mathrm{In}$. Moreover, the tumor can be imaged in 2-4 bed positions, enabled through the aforementioned dynamic list mode and the step-and-shoot $X Y Z$ stage. The good agreement with anatomic data is presented in the perpendicular slices through the SPECT image volume (Fig. 5).

\section{DISCUSSION}

We built a versatile SPECT system with an advanced user interface that can be used for performing molecular imaging in both mice and rats. The resolution of the U-SPECT-II system, in an FOV that can be set in any part of a mouse and for a large variety of imaging protocols, is unmatched by any previous PET or commercial SPECT system. With U-SPECT-II, it is possible to perform focused imaging, studying only a single organ of interest at extremely high 


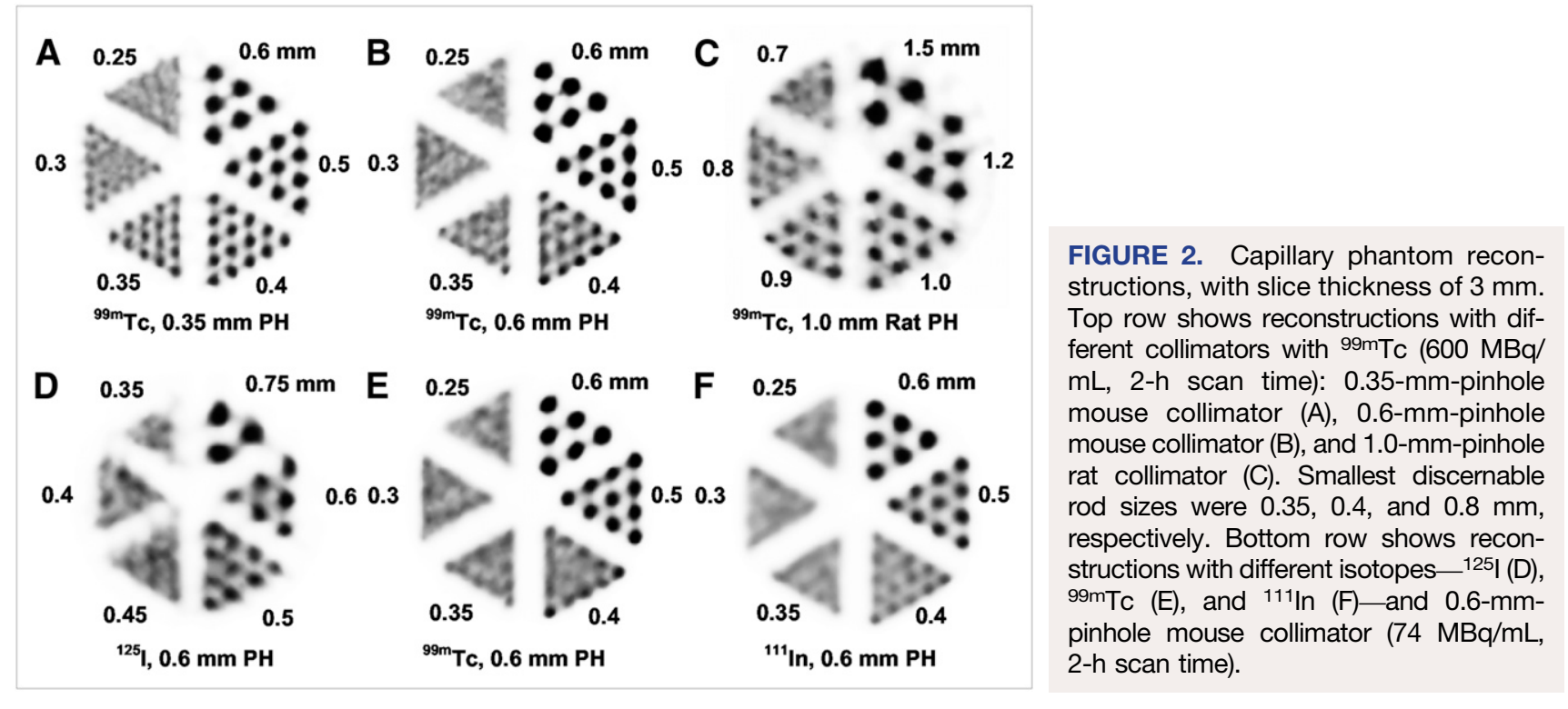

sensitivity and resolution and imaging larger volumes up to total-body imaging, with a single collimator tube. It is possible to improve significantly on the performance in specific cases with collimator tubes dedicated to specific tasks. For example, a dedicated collimator for extremities could have a much smaller diameter and the same number of pinholes. The resulting increase of sensitivity and magnification factor may improve the resolution to about $0.1 \mathrm{~mm}$ for legs and possibly to $0.25 \mathrm{~mm}$ for the mouse brain. Another example is a dedicated brain collimator for rats (21). However, the first improvements may be possible by selecting the right pinhole diameter for a specific study type.
The reconstructed image quality for short-scan time, lowdose studies was estimated by using only a part of the available counts from the list-mode data. An advantage of this approach is that no experimental differences, such as interanimal variability, influence the comparison, because the data being used are from the same acquisition. The simulation of lower activity by scaling the available listmode data underestimates the background component, which should remain constant over any acquisition time. Thus, a simulated low-count image will have a smaller background component than will an image that was actually acquired. For U-SPECT-II, the typical background counting rate in the

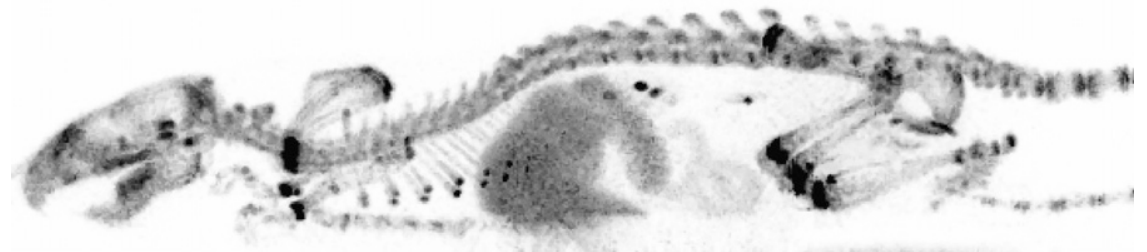

$100 \%=1,740 \mathrm{MBq}, 60 \mathrm{~min}$

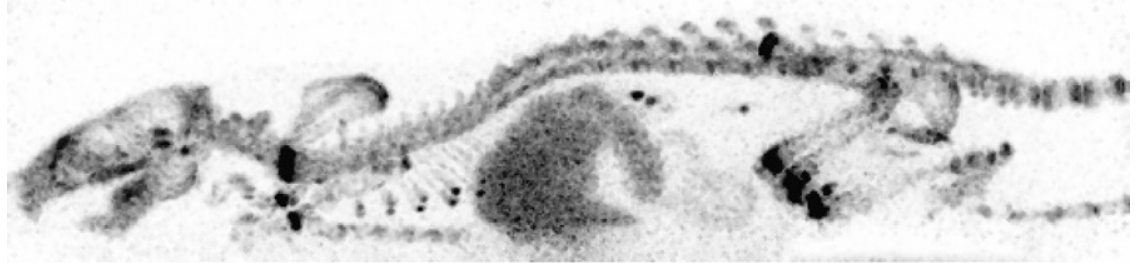

$10 \%$, equivalent to $1,740 \mathrm{MBq}, 6 \mathrm{~min}$, or $174 \mathrm{MBq}, 60 \mathrm{~min}$

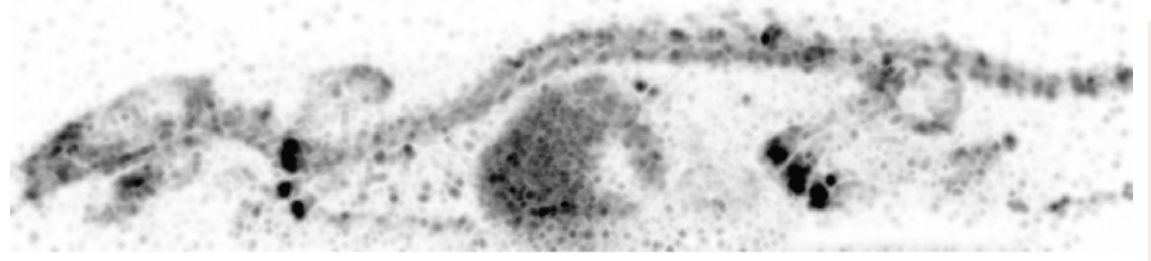

$1 \%$, equivalent to $1,740 \mathrm{MBq}, 36 \mathrm{~s}$, or $17 \mathrm{MBq}, 60 \mathrm{~min}$
FIGURE 3. Maximum-intensityprojection images of rat $99 \mathrm{mTc}-\mathrm{HDP}$ bone scan. Reconstruction used $100 \%$ down to $1 \%$ of available counts from list-mode data. Movie of gated reconstruction is shown in Supplemental Video 1. 
FIGURE 4. 99mTc-DMSA scan of mouse kidney (slice thickness, 0.375 $\mathrm{mm}$ ). Reconstruction was performed using 100\% (shown) and 10\% (Supplemental Fig. 1) of acquired counts. Image shows distribution of functioning kidney tissue. Such scans can be used to localize defects in parenchyma, to assess relative contribution of subcompartments of kidney.
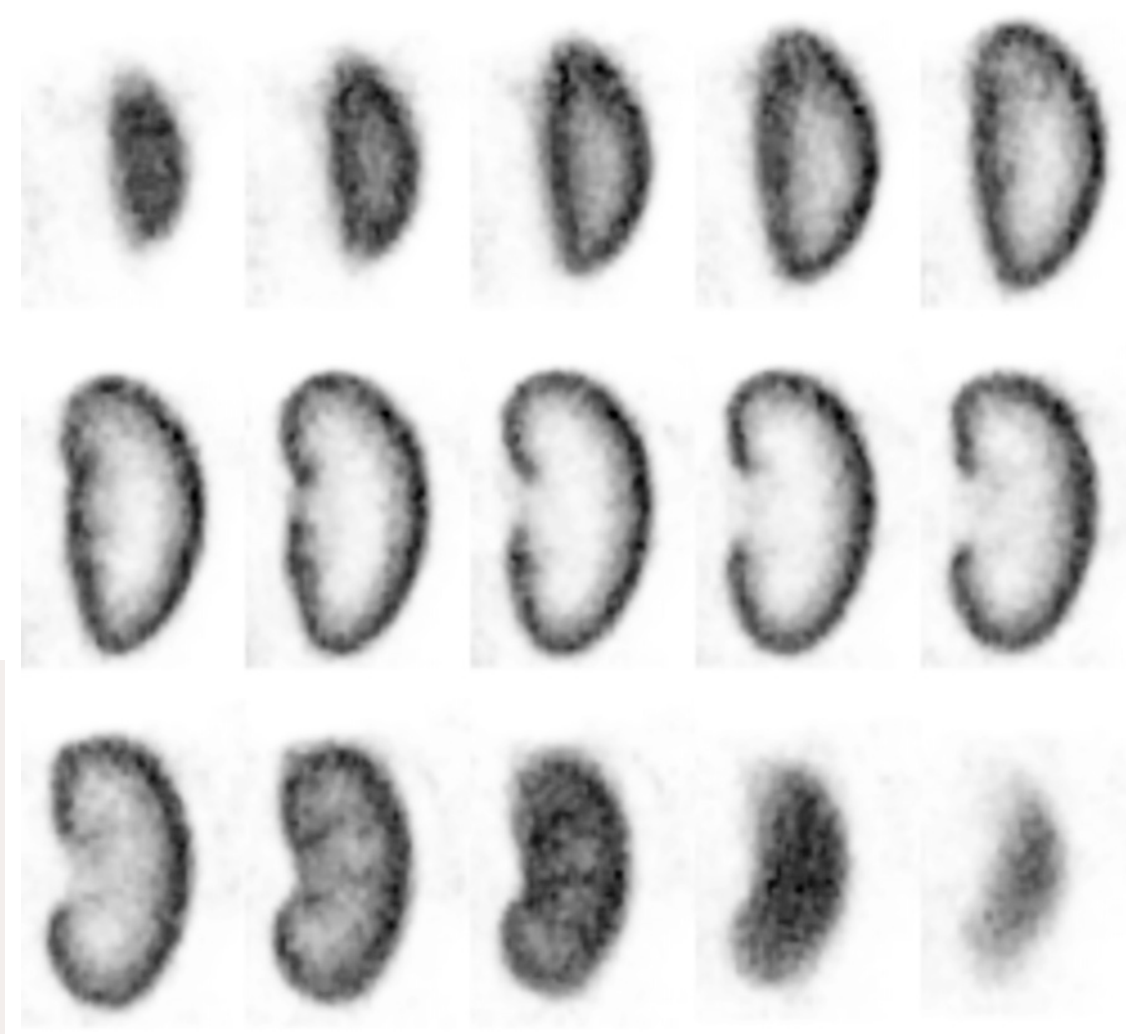

${ }^{99 \mathrm{~m}} \mathrm{Tc}$ window is $50 \mathrm{cps}$ for each of the $3 \gamma$-cameras, and a medium- to high-count animal acquisition produces 10,000 cps in that window. If the counting rate was reduced to $10 \%$ (i.e., 1,000 cps), we would be neglecting a background counting rate of about $5 \%$ of the total counts.

Today, the advantages and disadvantages of using overlapping versus nonoverlapping projections are actively being researched at several laboratories worldwide. Overlap increases system sensitivity, but one has to keep in mind that this increase is artificial; a significant amount of information per photon is lost because of the overlap. Therefore, sensitivity cannot be compared between systems with overlapping projections and systems with nonoverlapping projections. Moreover, overlap can lead to increasing reconstruction time due to the slow convergence of iterative algorithms for these cases and completely misplace the activity in the reconstruction when activity is present outside the area that is sampled (22).

\section{CONCLUSION}

U-SPECT-II is a new, ultra-high-resolution small-animal radiomolecular imaging system that allows the selection of

optimal collimators and FOV for different animals or imaging tasks. In addition, there is retrospective setting of movie frame length, gates, and energy channels. A volumetric resolution of $0.04 \mu \mathrm{L}$ has been obtained for ${ }^{99 \mathrm{~m}} \mathrm{Tc}$ with the highest-resolution collimators. Resolution was barely degraded for ${ }^{111}$ In and degraded by about $10 \%$ for ${ }^{125} \mathrm{I}$. The results for ${ }^{125}$ I show some additional distortion.

As a result, the system assesses multiple tracer dynamics in subcompartments of organs, as was illustrated by initial in vivo images and movies with submillimeter details of tracer uptake in rodent kidneys, tumors, and skeleton. Significant improvements in the image quality of U-SPECT can be expected when collimation parameters are optimized for specific tasks. High-resolution $\gamma$-detectors and collimators tailored to a specific application (e.g., joints, brain, or tumors) will play an important role in the future improvement of U-SPECT.

\section{ACKNOWLEDGMENTS}

We thank Norbert Gehéniau, Paul Hermans, Bert Westra, and Kees Ligtvoet for technical support and Jan Heemskerk

FIGURE 5. ${ }^{111}$ In-DTPA-14C5 mouse study. (A) Three orthogonal views of reconstructed data. (B) Photograph of tumor.
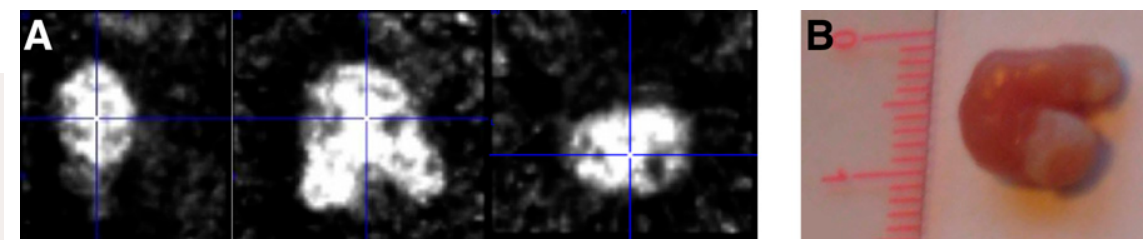
for proofreading the manuscript. This work was sponsored by the Netherlands Organization for Scientific Research (NWO), grant 917.36.335.

\section{REFERENCES}

1. King MA, Pretorius PH, Farncombe T, Beekman FJ. Introduction to the physics of molecular imaging with radioactive tracers in small animals. $J$ Cell Biochem. 2002;39(suppl):S221-S230.

2. Meikle SR, Kench P, Kassiou M, Banati RB. Small animal SPECT and its place in the matrix of molecular imaging technologies. Phys Med Biol. 2005;50:R45R61.

3. Beekman FJ, van der Have F. The pinhole: gateway to ultra-high resolution three-dimensional radionuclide imaging. Eur J Nucl Med Mol Imaging. 2007; 34:151-161.

4. Jaszczak RJ, Li J, Wang H, Zalutsky MR, Coleman RE. Pinhole collimation for ultra-high-resolution small-field-of-view SPECT. Phys Med Biol. 1994;39:425437.

5. McElroy DP, MacDonald LR, Beekman FJ, et al. Performance evaluation of A-SPECT: a high resolution desktop pinhole SPECT system for imaging small animals. IEEE Trans Nucl Sci. 2002;49:2139-2147.

6. Schramm NU, Ebel G, Engeland U, Schurrat T, Béhé M, Behr TM. Highresolution SPECT using multipinhole collimation. IEEE Trans Nucl Sci. 2003;50:315-320.

7. Walrand S, Jamar F, de Jong M, Pauwels S. Evaluation of novel whole-body high-resolution rodent SPECT (Linoview) based on direct acquisition of linogram projections. J Nucl Med. 2005;46:1872-1880.

8. Metzler SD, Jaszczak RJ, Patil NH, Vemulapalli S, Akabani G, Chin BB. Molecular imaging of small animals with a triple-head SPECT system using pinhole collimation. IEEE Trans Med Imaging. 2005;24:853-862.

9. Hesterman JY, Kupinski MA, Furenlid LR, Wilson DW, Barrett HH. The multimodule, multi-resolution system $\left(\mathrm{M}^{3} \mathrm{R}\right)$ : a novel small-animal SPECT system. Med Phys. 2007;34:987-993.
10. Liu Z, Kastis GA, Stevenson GD, et al. Quantitative analysis of acute myocardial infarct in rat hearts with ischemia-reperfusion using a high-resolution stationary SPECT system. J Nucl Med. 2002;43:933-939.

11. Furenlid LR, Wilson DW, Chen Y, et al. FastSPECT II: a second-generation high-resolution dynamic SPECT imager. IEEE Trans Nucl Sci. 2004;51:631-635.

12. Beekman FJ, van der Have F, Vastenhouw B, et al. U-SPECT-I: a novel system for sub-millimeter resolution tomography of radiolabeled molecules in mice. $J$ Nucl Med. 2005;46:1194-1200.

13. Funk T, Després P, Barber WC, Shah KS, Hasegawa BH. A multipinhole small animal SPECT system with submillimeter spatial resolution. Med Phys. 2006; 33:1259-1267.

14. Vastenhouw B, van der Have F, van der Linden AJA, et al. Movies of dopamine transporter occupancy with ultra-high resolution focusing pinhole SPECT. Mol Psych. 2007;12:984-987.

15. Kupinski MA, Barrett HH, eds. Small-Animal SPECT Imaging. New York, NY: Springer Science+Business Media Inc.; 2005.

16. van der Have F, Vastenhouw B, Rentmeester MCM, Beekman FJ. System calibration and statistical image reconstruction for ultra-high resolution stationary pinhole SPECT. IEEE Trans Med Imaging. 2008;27:960-971.

17. Vastenhouw B, Beekman FJ. Submillimeter total-body murine imaging with U-SPECT-I. J Nucl Med. 2007;48:487-493.

18. Chow PL, Stout DB, Komisopoulou E, Chatziioannou AF. A method of image registration for small animal, multi-modality imaging. Phys Med Biol. 2006; 51:379-390.

19. Beekman FJ, Hutton BF. Multi-modality imaging on track. Eur J Nucl Med Mol Imaging. 2007;34:1410-1414.

20. Beekman FJ. (WO/2007/043868) Radiation detection device, scintillation device and detection method, as well as multiple image-forming device. International application number PCT/NL2006/000513. April 19, 2007.

21. Beekman FJ, van der Have F, Vastenhouw B. Sub-half $\mathrm{mm}$ rat brain SPECT with a dedicated ultra-high resolution focusing pinhole collimator [abstract]. $\mathrm{J} \mathrm{Nucl}$ Med. 2008;49(suppl 1):401P.

22. Vunckx K, Suetens P, Nuyts J. Effect of overlapping projections on reconstruction image quality in multipinhole SPECT. IEEE Trans Med Imaging. 2008; 27:972-983. 